\title{
Ectopic mediastinal parathyroid carcinoma in a patient with end-stage renal disease
}

\author{
Seon-Ho Ahn ${ }^{1,2(i D}$, Jong Hwan Jung ${ }^{1,2}$ (i) \\ 'Division of Nephrology, Department of Internal Medicine, Wonkwang University Hospital, Wonkwang University School of Medicine, \\ Iksan, Republic of Korea \\ 2Institute of Wonkwang Medical Science, Wonkwang University School of Medicine, Iksan, Republic of Korea
}

A 30-year-old man receiving hemodialysis showed persistently high levels of serum intact parathyroid hormone (iPTH) and phosphate despite use of intravenous calcitriol. A parathyroid scan revealed increased uptake in both upper parathyroid glands (Fig. 1A). However, the lesions were thought to be parathyroid hyperplasia, not parathyroid adenoma. This is because the lesions, which showed increased uptake, did not appear to be nodular on the ultrasonography. Cinacalcet and calcitriol were used simultaneously to treat the tertiary hyperparathyroidism. After 8 months, a follow-up scan was performed, which showed increased uptake in both upper parathyroid glands. Also, hot lesions in the left lower parathyroid gland and right upper paratracheal area of the mediastinum newly developed (Fig. 1B). Computed tomography (CT) revealed two nodular lesions in the mediastinum
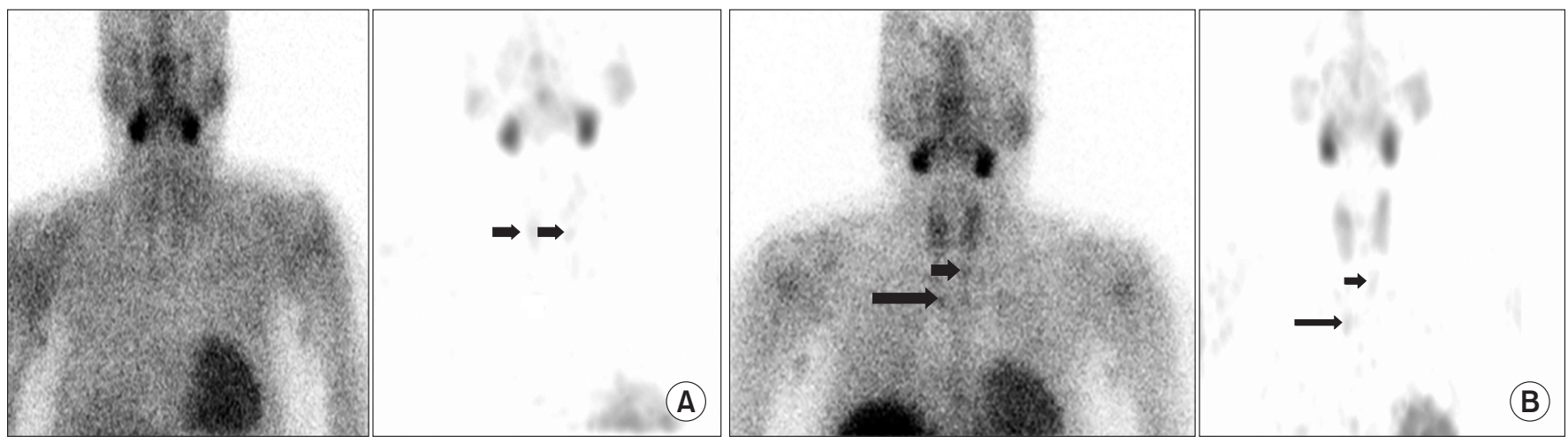

Figure 1. (A) The first parathyroid scan with technetium-99m tetrofosmin shows increased uptake in both upper parathyroid glands (arrows). (B) A follow-up parathyroid scan with technetium-99m tetrofosmin shows hot lesions newly developed in the left lower parathyroid gland and right upper paratracheal area of the superior mediastinum (arrows).

Received February 5, 2020; Revised March 18, 2020; Accepted March 21, 2020

Edited by Jeonghwan Lee, Seoul National University, Seoul, Republic of Korea

Correspondence: Jong Hwan Jung

Division of Nephrology, Department of Internal Medicine, Wonkwang University Hospital, Wonkwang University School of Medicine, 895 Muwang-ro, Iksan 54538, Republic of Korea. E-mail: chjho502@gmail.com

Copyright (C) 2020 by The Korean Society of Nephrology

(a) This is an open-access article distributed under the terms of the Creative Commons Attribution Non-Commercial License (http://creativecommons.org/ licenses/by-nc-nd/4.0/), which permits unrestricted non-commercial use, distribution, and reproduction in any medium, provided the original work is properly cited. 


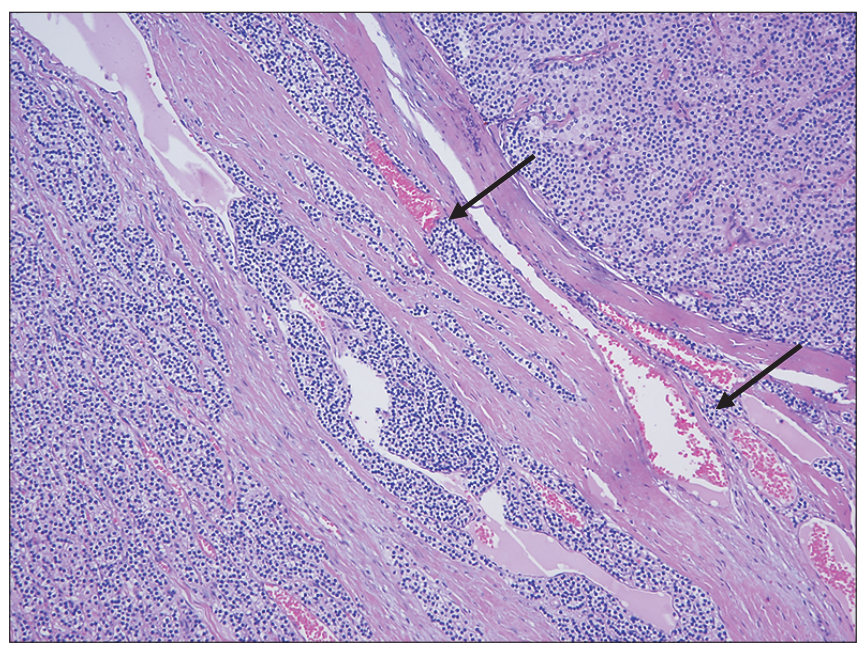

Figure 2. The pathological finding of the mediastinal masses reveals parathyroid carcinoma with early capsular invasion (arrows) (hematoxylin-eosin staining; original magnification, $\times 100$ ).

along with hyperplasia in both upper and left lower parathyroid glands. The patient received an operation for removal and diagnosis of the mediastinal masses. The preoperative data were as follows: serum calcium, 10.4 $\mathrm{mg} / \mathrm{dL}$ and serum iPTH, $1,150 \mathrm{pg} / \mathrm{mL}$. The mediastinal masses were histologically compatible with parathyroid carcinoma with vascular involvement (Fig. 2). After the operation, the serum calcium level decreased, but serum iPTH level did not decrease. Oral calcitriol was continued, and an 18 F-fluorodeoxyglucose positron-emission tomography CT scan for distant metastases of the parathyroid carcinoma was performed. Metastatic lesions associated with the parathyroid carcinoma were not found. Total parathyroidectomy for tertiary hyperparathyroidism was performed. After total parathyroidectomy, only lesions with adenomatous hyperplasia on both upper and lower parathyroid glands were detected, and the serum iPTH level decreased to normal level and serum calcium was maintained within the normal range with oral cal- citriol treatment.

Parathyroid carcinoma is a rare cancer found in less than $1 \%$ of patients with primary hyperparathyroidism. Also, it may rarely develop even in the setting of tertiary hyperparathyroidism, where it may be difficult to diagnose without confirmative surgery. Thus, an ectopic mediastinal parathyroid carcinoma under the same conditions can be underdiagnosed or misdiagnosed. Although the causal relationship between ectopic parathyroid carcinoma and parathyroid hyperplasia under tertiary hyperparathyroidism is not clear, ectopic parathyroid carcinoma may develop along with parathyroid hyperplasia if the parathyroid glands are persistently stimulated by tertiary hyperparathyroidism. Thus, patients with tertiary hyperparathyroidism should be followed up with a CT scan series to detect any new lesions that may occur, even if the first scan only shows increased uptake in the parathyroid glands.

\section{Conflicts of interest}

All authors have no conflicts of interest to declare.

\section{Funding}

This work was supported by Wonkwang University in 2020.

\section{Authors' contributions}

Seon-Ho Ahn participated in the data collection and the study design and coordination and helped to draft the manuscript. Jong Hwan Jung participated in the conception, analysis, and interpretation of data, and wrote the manuscript and finally reviewed the article. All authors read and approved the final manuscript. 